\title{
Deserción universitaria en Ingeniería en Sistemas Computacionales. El caso de la Universidad Politécnica Metropolitana de Puebla
}

\author{
University Dropout in Computer Systems Engineering. The case of the \\ Universidad Politecnica Metropolitana de Puebla
}

\author{
Elvia Bautista Pérez \\ Benemérita Universidad Autónoma de Puebla, Facultad de Filosofía y Letras \\ elviabautistaperez@yahoo.com.mx
}

\section{Resumen}

La disciplina en ingeniería en sistemas computacionales es un campo amplio de investigar debido a que es una carrera nueva y multidisciplinaria. Sin embargo existe un problema latente: el índice de deserción. En el periodo de Enero a Abril de 2013 al 2015 en la Universidad Politécnica Metropolitana de Puebla se registró un $60 \%$ de desertores; para encontrar las causas a este problema, de deserción se realizaran la medición de algunas variables que influyen en dicha problemática como: factores económicos, reprobación y plan de estudios. Debido a que los factores de deserción pueden ser multifactoriales, se aplicó un instrumento que permitió conocer las causas de la deserción. Para poder darles solución y ayudar a todos los involucrados en dicha situación. Los resultados se realizaron con los datos obtenidos del cuestionario aplicados a 11 desertores, todo esto expuesto en las gráficas que permitieron un mejor entendimiento de la información recopilada.

\section{Palabras claves}

Deserción, factor económico, reprobación, plan de estudios, Ingeniería en Sistemas Computacionales.

\begin{abstract}
Computer systems engineering discipline is a broad field of research because it is a new and multidisciplinary career. However, there is a latent problem: the dropout rate. In the January-April 2013-2015 period at the Universidad Politecnica Metropolitana de Puebla 60\% of dropouts were registered; To find the causes of this problem, several variables were measured: Economic factors, failing rate and study plan. Because desertion factors can be multifactorial, an instrument was applied that allowed to know the causes of the desertion. To be able to help all those involved in this situation. The results were obtained with the data obtained from the survey applied to 11 deserters, all of this is shown in the graphs that allowed a better understanding of the collected information.
\end{abstract}

\section{Keywords}

Dropout, economic factor, failure, study plan, Computer Systems Engineering. 


\section{Introducción}

La importancia del estudio de deserción universitaria es trascendental debido a las altas cifra de deserción, reprobación y falta de orientación vocacional en los estudiantes de ing. en sistemas computacionales, varios estudios en el tema consideran que existen diversos factores que llevan a los alumnos a abandonar sus estudios universitarios.

La presente investigación se centra en la generación 2013, donde se tomó en cuenta la carrera de ing. sistemas computacionales de la Universidad Politécnica Metropolitana de Puebla, para ubicar principalmente los factores y consecuencias que ocasiona la deserción escolar universitaria.

Es importante señalar que la deserción universitaria se agrava cuando las instituciones educativas no readmiten a sus repetidores; ante esta situación los estudiantes deben buscar otra institución para dar continuidad a su formación, con frecuencia alejados de sus zonas de residencia $\mathrm{y}$ por razones económicas terminan por abandonar el sistema educativo; la universidad al expulsar a estos jóvenes los convierte en desertores, poniendo en duda su futuro, ya que se configuran a partir de este momento trayectoria vitales $y$ laborales asignadas por la frustración y la precariedad por falta de una formación académica que responda a las exigencias de los actuales mercados laborales.

Desde el punto de vista de varios autores, se mostraran algunas definiciones con el objetivo de dar una idea de los diversos estudios que existen respecto al fenómeno de deserción. Una de ellas, en la que de acuerdo a Tinto (2002) define el abandono estudiantil como el fracaso para completar un determinado curso o alcanzar una meta deseada. Acorde con la deserción en el ámbito superior influye de manera notable una serie de factores como: problemas para el aprendizaje, económicos y familiares que son relativos al propio estudiante y que determinan su falta de interés o de motivación para acudir a diario a clase.

\section{Causas de la deserción}

La deserción estudiantil que impacta en los indicadores institucionales es un problema que se caracteriza en muchas instituciones de educación pública y privada. Por otro parte existe un desinterés en los estudiantes durante su paso por una carrera universitaria por lo que puede ser tan elevado que nos lleve a un abandono de las aulas.

En un análisis realizado por Tinto (2001) informa que el $50 \%$ de todas las deserciones ocurren antes del segundo año y que el $40 \%$ de todos los estudiantes no logran graduarse. Otro rasgo importante de la deserción se ha detectado que un estudiante que llega a la universidad con altas expectativas personales manifiesta una desmotivación debido a que no era lo que ellos esperaban.

Con respecto a la deserción estudiantil puede entenderse simplemente como la disolución del vínculo a través de la matrícula académica, por cualquier causa ya sea por parte del estudiante o de la universidad. Esta, tiene graves efectos financieros, académicos y sociales para ambos. Rodríguez y Hernández (2008).

"Habría que mencionar que las ingenierías buscan describir el mundo que nos rodea. Se puede decir que son los cimientos de la tecnología actual y una parte considerable del progreso de la humanidad. Una ingeniería tiene el objetivo de desarrollar un pensamiento lógico en los alumnos para resolver problemas con ingenio. Se dicen que son ingenierías duras debido a los altos índices de reprobación en materias relacionadas con matemáticas" De la Cruz (2008).

Por último la Ingeniería en Sistemas computacionales presenta desde el año 2010 una deserción escolar alta, en donde ingresan18 alumnos en cada ciclo escolar, 
desde el primer cuatrimestre empiezan a desertar por los siguientes conceptos: factores económicos, reprobación y plan de estudios.

\section{Fundamento teórico}

Una de las teoría más extensamente aceptada entre la comunidad académica es la teoría de exclusión escolar que expuso Tinto.

Tinto (2002) expuso que el grado en que el estudiante se integre al mundo académico y social de una institución determinará si realmente un estudiante se mantiene matriculado o no en la universidad. Su teoría establece que los estudiantes entran en la universidad con varias características individuales que incluyen la familia y las características de trasfondo comunitario, el nivel educativo de los padres, status social, atributos individuales, capacidad, raza, carrera , género, habilidades intelectual, social, recursos financieros, la disposición, motivaciones, intelecto, preferencias políticas, la experiencia educativa en la escuela, registro del logro académico.

\section{Problemática actual en la Ingeniería sistemas computacionales}

La Universidad Politécnica Metropolitana de Puebla (UPMP) surge en el año 2010 con la visión de ofertar planes de estudio de calidad en un corto tiempo, ubicada en la Calle Popocatépetl s/n de la colonia tres cerritos, El modelo educativo basado en competencias es el que actualmente se implementa para la transmisión de conocimientos. Entre las carreras que oferta se encuentran: Ingeniería en Biotecnología, Ingeniería en Sistemas Computacionales y Licenciatura en Administración y Gestión de Pequeñas y Medianas Empresas. Por otro lado, la Ingeniería en Sistemas computacionales es una de las carreras más demandadas debido a su perfil multidisciplinario. Sin embargo, la demanda académica es fuerte y requiere de dedicación para aprender campos distintos de la ingeniería.

Unos de los problemas que se tiene es el índice de deserción elevado en la ingeniería en sistemas computacionales. En el periodo de 2013 al 2015, se registró un 60\% de deserción dentro de la carrera mencionada anteriormente, la ingeniería en sistemas computacionales presentó el mayor índice de reprobación en la UPMP. Las causas en una carrera multidisciplinar son aún desconocidas debido a que existen materias con tópicos muy diversos y es difícil establecer un punto de partida.

Varias investigaciones sobre deserción en carreras de ingeniería han sido abordadas anteriormente. Sin embargo, no se encontraron investigaciones relacionadas con el campo multidisciplinar de la ingeniería en Sistemas computacionales. Es por esto que el enfoque del trabajo propuesto tiene como objetivo determinar los factores que inciden en la deserción escolar de los estudiantes de Ingeniería en Sistemas computacionales de la Universidad Politécnica Metropolitana de Puebla. Para esto se plantea las siguientes preguntas:

1. ¿Cuáles son los factores de índole institucional que inciden en la deserción universitaria de los alumnos de ingeniería en sistemas computacionales?

2. ¿Cuáles son los factores más relevantes que influyen en la deserción universitaria de los estudiantes de la ingeniería en sistemas computacionales de la Universidad Politécnica Metropolitana de Puebla?

3. ¿Cuáles son los factores relacionados con el comportamiento académico que influyen en la deserción universitaria de los estudiantes de ingeniería en sistemas computacionales? 


\section{Metodología}

Este estudio se orienta bajo los parámetros de la investigación cuantitativa, tomando como referencia las características de la situación del problema factores que contribuyen en la deserción Universitaria en la Ingeniería en Sistemas Computacionales El caso de la Universidad Politécnica Metropolitana de Puebla, se enfoca hacia un tipo de investigación no experimental de tipo descriptiva pues se recolectaron datos o componentes sobre los diferentes factores que contribuyen a la deserción y se realizó un análisis y medición de los mismos.

La población la constituyeron alumnos desertores en ingeniería en sistemas computacionales de la universidad politécnica metropolitana de puebla se trabajó con un total de 11 desertores.

Se aplicó una prueba piloto aleatoria simple, seleccionando a los alumnos de los listados de la generación 2013 que proporciono el departamento de servicios escolares, los cuales señalaban un total de 18 alumnos de la ingeniería en sistemas computacionales 11 desertaron y 7 permanecen en la institución. Estos listados incluían los números telefónicos y domicilio de los alumnos. De los 11 desertores solo se encuestaron 10 ya que se tuvo dificultad localizar a uno de ellos.

\section{Descripción de los sujetos que se encuestaron}

Los alumnos que se encuestaron fueron un total de 11 desertores, dentro del grupo de los desertores 5 pertenecen al género femenino y 6 al masculino, el rango de edad de inclusión será de 18 a 35 años de edad.

\section{Descripción del instrumento}

El diseño del instrumento aplicado a los alumnos que se utilizó para la medición de algunas variables que provocan la deserción estudiantil en la UPMP, consto 40 preguntas en donde solo se consideran 3 variables como: deserción, reprobación y plan de estudios; estas variables se consideraron tomando en cuenta algunas característica personales de los sujetos que conforman nuestra muestra.

Para la elaboración del instrumento se realizaron los siguientes pasos:

Se realizó una revisión de la definición conceptual de cada variable.

Se realizó una revisión de indicadores por variables.

Se realizaron indicaciones para el llenado para el llenado de la encuesta

\section{Resultados}

Con el propósito de cumplir con los objetivos planeados en este trabajo se llevaron a cabo el análisis de los resultados se estudiaron los factores sociodemográficos, factores económico, orientación vocacional, aspectos familiares y personales, factores laborales, factores de salud, adaptación universitaria, factor motivacional, factores académicos, técnicas de estudio, didácticas docentes y plan de estudios con el fin de conocer los factores que influyen en la deserción.

Datos sobre los aspectos sociodemográficos Sobre los estudios demográficos se observa que 3 son hombres que corresponde $27 \%$ son hombres y 8 son mujeres que corresponde al $73 \%$ las edades de los sujetos van de los 18 a 35 años La media de edad de los desertores es de $\operatorname{los} 25$ años.

Aspectos relacionados con los factores económicos

Respecto a los factores económicos se identificaron las causas por las fueron dados de baja, con un $40 \%$ el tipo de factores que motivaron su retiro de los estudios universitarios con un 60\%.Las aspectos fueron: los jóvenes trabajan y estudian al mismo tiempo, existen pocas facilidades para realizar sus actividades académicas en el trabajo y compromisos familiares. 
Aspectos relacionados con la orientación vocacional

En los resultados se observa los estudiantes que desertaron se debe a la falta de orientación vocacional, se les pregunto a los sujetos si al momento previo de ingresar en la carrera recibió orientación vocacional un 30\% recibió orientación vocacional el resto corresponde al $70 \%$ no recibió orientación alguna.

\section{Motivos para abandonar la carrera}

Los sujetos que desertaron de la carrera de ingeniería en sistemas computacionales se les pregunto cuáles fueron las causas por la que abandonaron la carrera y las más mencionadas fueron: No me gustaba la carrera con un $20 \%$, no me sentí identificado/a con la carrera $40 \%$, por embarazo un $20 \%$ y problemas económicos con un $20 \%$.

\section{Aspectos familiares y personales}

Respecto a los factores familiares se identificó el entorno familiar o social como causante de la deserción y se encontró que el entorno familiar si está de acuerdo que la en la deserción estudiantil se debe a factores familiares con un $40 \%$, otros están de acuerdo con un $20 \%$, en desacuerdo con un $20 \%$ y totalmente en desacuerdo con un $20 \%$.

\section{Factores laborales}

Respecto a este factor se identificaron la siguiente pregunta estudia al mismo tiempo que trabajaba el $60 \%$ respondió que si trabajaba, la otra parte no trabaja con un $40 \%$.

\section{Factores de salud}

En este factor se le pregunto con qué frecuencia tenían problemas de salud que les impidiera cumplir con sus actividades escolares el $20 \%$ contesto nunca había tenido alguna molestia, el $35 \%$ contesto que rara vez tenía alguna molestia, el $20 \%$ contesto que una vez a la semana habían tenido algún problema de salud, el $10 \%$ contesto dos o tres veces a la semana había tenido algún problema de salud, $10 \%$ respondió que casi diario tenían algún problema de salud con el $5 \%$

\section{Adaptación universitaria}

En la adaptación universitaria se les preguntó las 3 razones por la cual eligió la universidad politécnica metropolitana de puebla, el $10 \%$ Por los planes de estudio, 10\%por la carreras son innovadoras, $10 \%$ por las instalaciones, $25 \%$ por la modalidad de titulación, $30 \%$ por que terminan la carrera en tres años y medio, $5 \%$ las carreras que ofrecen tienen amplio campo laborar, $5 \%$ por la movilidad estudiantil, $5 \%$ por las becas que ofrece la institución.

\section{Factor motivacional}

En este factor se les pregunto cuando iniciaron el cuatrimestre $¿$ Qué esperaban de la carrera? el $30 \%$ mucha práctica, $20 \%$ que los integraran a proyectos con compañeros de cuatrimestre más avanzados, $10 \%$ querían asistir a congresos, empresas y viaje de prácticas, $20 \%$ que los tomaran en cuenta para las actividades de la coordinación, 20\% que les dejaran aportar para la carrera con nuevas ideas.

\section{Factores académicos}

En este factor se les pregunto sobre su rendimiento académico antes de desertar fue, un $20 \%$ respondió que fue excelente, $30 \%$ su rendimiento académico era bueno, 30\% su desempeño académico era regular, $10 \%$ respondió como un rendimiento deficiente, por último el $10 \%$ respondió que su rendimiento académico fue muy deficiente.

\section{Técnicas de estudio}

Se les realizo la siguiente pregunta con base a tu percepción que tan apto te consideras para estudiar solo el 30\% que era excelente 
estudiando solo, sobre las habilidades matemáticas el 25\% respondió ser bueno en matemáticas, para redactar trabajos en español el 15\% dijo ser bueno en redacción, uso del lenguaje oral $15 \%$ era bueno en el lenguaje oral y por ultimo 15\% n comprendía un texto en inglés.

\section{Didácticas del docente}

En este apartado se les hizo la siguiente pregunta la calidad de los docentes que le impartieron clases fue, $30 \%$ manifestó que eran excelentes docentes,30\% dijeron que eran buenos maestros, $20 \%$ calificaron a los docentes como regular, 10\% consideraron deficientes a sus docentes y el resto10\% creían que sus maestros eran muy deficientes.

\section{Plan de estudios}

Respecto al plan de estudio se les realizo la siguiente pregunta en plan de estudios responde a tus expectativas personales $10 \%$ considera que el plan de estudio si cumples con sus expectativas personales 30\% no encontraron compatibilidad en el programa de estudio, 30\%los cursos que integran el plan de estudios no son importantes para su formación profesional 30\% porque no existe una relación directa entre los cursos del plan de estudios y el campo laboral.

\section{Conclusiones}

Como resultado los factores por lo que están desertando los estudiantes de sistemas computacionales de la Universidad Politécnica Metropolitana de Puebla se debe aspectos económicos, la institución no proporciona las becas adecuadas, estímulos académicos para los estudiantes y tiene como consecuencia que los jóvenes abandonen sus estudios universitarios.

Otro aspecto importante de la deserción en sistemas computacionales se debe a la reprobación estudiantil, hasta el momento la institución no ha tomado las medidas pertinentes, para reducir el número de reprobados.

En vista que tenemos la falta de pertinencia de los planes y programas de estudio, los alumnos están desertando por que no encuentran congruencia entre los planes de estudio y con el campo laboral, es necesario que la universidad realice una revisión de sus planes y programas, ya que desde esta perspectiva se podría reducir la deserción.

Considerando que es sumamente importante poner más atención en los jóvenes desertores e implementar un programa de retención en la Universidad Politécnica Metropolitana de puebla, difundir las becas estudiantiles e implementar más programas de estímulos económicos para disminuir esta problemática, motivar a los jóvenes a no dejar de estudiar y hacer una reestructura de los planes y programas de estudio.

Dado que los alumnos que reciben orientación vocacional son los que permanecen en la carrera, los que no recibieron orientación vocacional tienen un alto índice deserción. Esta situación se considera de suma importancia que la universidad realice un proceso de selección más específico para que solamente ingresen alumnos que tengan las aptitudes necesarias para el desarrollo de la profesión.

Por último es necesario una revisión del plan de estudios de esta manera se evitara la inversión de recursos humanos y económicos en alumnos que por sus escasas aptitudes para la carrera, tendrán más posibilidad de desertar, ahorrando recursos a la familia, la comunidad, la universidad y al propio país.

\section{Referencias}

ANUIES (2007); Retención y deserción en un grupo de instituciones mexicanas de educación superior. Colección Biblioteca de la Educación Superior, México.

Bourdieu, P y Passeron J.C.(1970); La reproducción Elementos para una 
teoría del sistema de enseñanza. Fontamara, Primera edición en 1995, primera reimpresión 2009, México.

Bourdieu, P. (1997). Capital cultural, escuela y espacio social. Siglo xxi.

Covo, Milena. Algunas condiciones no académicas de la deserción. En Nuevas perspectivas críticas de la Universidad. México. Cuadernos del CESU No. 16. UNAM. México. 1989.

Cruz López, Y. \& Cruz López, A. K. (2008). La educación superior en México. Tendencias y desafíos. Avaliação: Revista da Avaliação da Educación Superior, 13(2) 293-311. Recuperado de http://www.redalyc.org/articulo.oa?i $\mathrm{d}=219114873004$

México. (2013) Panorama de la educación. OCDE. Recuperado de. http://www.oecd.org/edu/Mexico_EA G2013\%20Country\%20note\%20(ESP J.pdf
OCDE, (2007). Estudio Panorama de la Educación en el 2006. Consultado el 15 de febrero de 2011 en: http://www.oecd.org/dataoecd/42/5 4/43638848.pdf

Perrenoud, P. (1990). La construcción del éxito y del fracaso escolar: hacia un análisis del éxito, del fracaso y de las desigualdades como realidades construidas por el sistema escolar. Ediciones Morata.

Soria-Barreto, K., \& Zúñiga-Jara, S. (2014). Aspectos Determinantes del Éxito Académico de Estudiantes Universitarios. Formación universitaria, 7(5), 41-50.

Tinto, V. (1989). Definir la deserción: una cuestión de perspectiva. Revista de Educación Superior, 71, 33-51.

Unesco, I. E. S. A. L. C. (2006). Repitencia y Deserción Universitaria en América Latina. 
Bautista Pérez, E. 